\title{
Saúde mental e níveis de atividade física de crianças escolares
}

\author{
Gabrielle Cerqueira da Silva ${ }^{a}$, Jorge Lopes Cavalcante Neto ${ }^{a, b}$

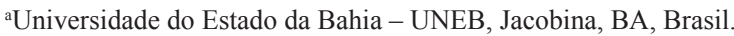

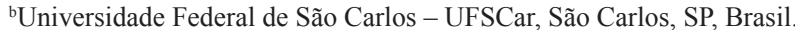

\begin{abstract}
Resumo: Introdução: A prática regular de atividade física é fundamental para a saúde das crianças, tendo sido apontada como fator de proteção para problemas de cunho mental na idade escolar. Objetivo: Verificar a relação entre problemas de saúde mental e os níveis de atividade física em crianças escolares da cidade de Jacobina-BA. Método: Amostra composta por 55 escolares, de 8 a 10 anos e seus pais, que participaram como informantes secundários do estudo. Utilizou-se o Child Behavior Checklist (CBCL) para avaliação dos problemas de saúde mental dos escolares, o Physical Activity Questionnaire for Older Children (PAQ-C) para avaliação dos níveis de atividade física dos sujeitos e um questionário sociodemográfico. Resultados: A maioria das crianças foi classificada como sedentária $(80 \%)$ e apenas $7,3 \%$ da amostra apresentou rastreamento positivo para transtorno mental. Não foi encontrada associação significativa entre o transtorno mental e os níveis de atividade física entre o grupo, nem entre essas variáveis e as características sociodemográficas das crianças. Observou-se que as meninas estavam mais sedentárias que os meninos, com diferença significativa ( $<<0,01$ ). Conclusão: Apesar da baixa prevalência de problemas de saúde mental entre os escolares e a não associação com os níveis de atividade física, faz-se necessária atenção especial com esse público, visando fortalecer a atividade física como fator protetor à saúde mental das crianças, com investimentos em ações que visem ao incentivo de prática regular de atividade física, aliando família e escola. Estudos com um número maior de amostras precisam ser conduzidos para ser analisado com cautela os seus achados.
\end{abstract}

Palavras-chave: Crianças, Saúde Mental, Atividade Motora.

\section{Mental health and physical activity levels of school children}

\begin{abstract}
Introduction: The regular practice of physical activity is fundamental to the health of children, it has been cited as factor of protection for mental disorders in school age. Objective: To verify the relation between mental health and physical activity levels in schoolchildren of the city Jacobina, Bahia. Method: Sample composed of 55 students between the ages 08 to 10 and their parents, who participated as secondary informants in this study. The Child Behavior Checklist (CBCL) was used for evaluation of mental health problems of the schoolchildren, Physical Activity Questionnaire for Older Children (PAQ-C), for evaluation of the physical activity levels of the subject, and a social-demographic questionnaire. Results: Most children were classified as sedentary (80\%) and only $7.3 \%$ of the sample showed positive for trace of mental disorder. No significant association was found between mental disorders and physical activity levels among the group, or between these variables and socio-demographic characteristics of children. It was observed that the girls were more sedentary than boys, with significant difference $(\mathrm{p}<0.01)$. Conclusion: Despite the low prevalence of mental health problems among schoolchildren and the non-association with physical activity levels, special attention is necessary with this audience, aiming to strengthen physical activity as a protective factor for children's mental health, with investments in actions aimed at the encouragement of regular practice of physical activity, combining family and school. Studies with a larger number of samples need to be conducted and its findings must be thoroughly analyzed.
\end{abstract}

Keywords: Children, Mental Health, Motor Activity.

Autor para correspondência: Jorge Lopes Cavalcante Neto, Av. J. J. Seabra, s/n, Bairro Estação, CEP 44700-000, Jacobina, BA, Brasil, e-mail: jorgelcneto@hotmail.com

Recebido em Mar. 17, 2016; $1^{\text {a }}$ Revisão em Maio 21, 2016; 2 ${ }^{\mathrm{a}}$ Revisão em Jul. 31, 2016; Aceito em Ago. 4, 2016. 


\section{Introdução}

Os casos de problemas mentais têm afetado os indivíduos, independentemente de sexo e idade. Estima-se que milhóes de crianças apresentam sintomas psicopatológicos, não são identificadas e não recebem atendimento (RAMIRES et al., 2009a).

Os primeiros sinais de transtornos mentais e comportamentais aparecem no período da infância. Entretanto, existe uma maior dificuldade de se chegar a um diagnóstico patológico nessa fase do desenvolvimento, e isso acaba comprometendo o desenvolvimento da criança na família, escola e sociedade (AVANCI; ASSIS; PESCE, 2008).

Os resultados negativos no desenvolvimento e comportamento da criança são produzidos pela combinaçâo de fatores de risco genéticos, biológicos, psicológicos e ambientais, envolvendo interaçóes complexas entre eles. Desses fatores, os mais frequentemente associados à saúde mental são o ambiente social e o psicológico, os quais influenciam mais do que as características intrínsecas do indivíduo. Além disso, o efeito acumulativo de risco é mais importante na determinação de problemas emocionais da criança do que a presença de um estressor único (HALPERN; FIGUEIRAS, 2004).

Segundo a Organização Mundial da Saúde (2001), há vários fatores de risco para o desenvolvimento de perturbaçóes mentais, principalmente no período da infância. A exemplo, existem os fatores sociais, como a urbanização descontrolada, a pobreza e a rápida transformação tecnológica. Outro fator relevante é a relaçáo da criança com os pais ou outros cuidadores durante a infância, pois uma criança privada de um envolvimento afetivo tem mais probabilidades de sofrer perturbaçóes de comportamento, seja na infância, adolescência ou ao longo da vida adulta. Além disso, o sexo, a idade, os conflitos e os traumas pessoais, as doenças físicas e o ambiente familiar e social determinam a prevalência e a manifestação das questôes citadas (ASBAHR; TIWARI; KENDALL, 2007; CONNOLLY; BERNSTEIN, 2007).

As manifestaçôes desses problemas mentais muitas vezes ocorrem em conjunto no mesmo indivíduo, que comumente desenvolve transtornos de ansiedade combinados com perturbaçóes depressivas (ASBAHR; TIWARI; KENDALL, 2007; CONNOLLY; BERNSTEIN, 2007). Para todas as pessoas, a saúde mental, a saúde física e a social estão estreitamente ligadas. Assim, compreende-se que a saúde mental é indispensável para o bem-estar geral dos indivíduos e da sociedade (ORGANIZAÇÃO..., 2001).
De acordo com a Organização Mundial da Saúde (2001), a saúde mental e a saúde física são dois elementos da vida que estão totalmente entrelaçados e interdependentes, pois muitas perturbaçôes mentais, comportamentais e doenças físicas resultam de alguns fatores biológicos, psicológicos e sociais.

Como a saúde mental e a saúde física são elementos que estão interligados, a prática regular da atividade física é uma forma de proporcionar vários benefícios à saúde, é uma efetiva prevenção à ocorrência de doenças futuras. Assim, em relação às crianças, a atividade física também desempenha papel fundamental sobre a condição física, psicológica e mental (SILVA; COSTA, 2011).

Conforme o exposto, vale ressaltar que a atividade física pode ser utilizada para intensificar os fatores de proteçáo aos problemas de saúde mental aos quais as crianças estão sujeitas (NOVAES, 2005). A literatura vem reunindo evidências importantes que direcionam a prática regular de atividade física como fator protetor aos problemas de saúde mental durante a infância e a adolescência (MEARS; JAGO, 2016).

Assim, percebe-se que cada vez mais são necessários ambientes de cuidados que atendam às necessidades das crianças, incentivando-as à prática de atividades físicas como um requisito básico ao desenvolvimento, e esse é um desafio a ser superado pela família, escola e comunidade (SILVA; COSTA, 2011).

Ainda existe uma falta de interesse em relação à saúde mental durante a infância, o que pode acarretar consequências negativas no decorrer do desenvolvimento desses indivíduos, já que poucas pessoas se importam em saber como está a saúde mental de uma criança, e isso acaba afetando sua capacidade produtiva e a inserçáo social quando adultos, e também irá refletir-se no nível social e econômico das coletividades (RAMIRES et al., 2009b).

A atenção à saúde mental das crianças está sendo cada vez mais necessária. Porém, a falta de serviços e especialistas na área é preocupante. Esse fato contribuiu para que os profissionais de saúde, de maneira geral, tenham dificuldade em atender crianças com algum tipo de comprometimento emocional. Além disso, para os poucos serviços oferecidos, as filas de espera são longas e nem sempre as crianças recebem assistência adequada (XIMENES; PESCE, 2009).

Dessa forma, é necessário planejar intervençôes visando à saúde mental da criança e do adolescente, incrementando e potencializando serviços de atendimento nessa área, para que seja feita uma melhor análise da complexidade das situaçóes adversas e de 
risco. São estratégias fundamentais tanto no âmbito de prevenção dos problemas, como de intervenção (RAMIRES et al., 2009b).

Vale ressaltar que a participação das crianças em projetos sociais pode ser considerada fator protetivo da saúde mental, assim, torna-se fundamental a implementação de políticas, serviços de saúde mental e práticas preventivas nessa área voltadas para a população de crianças e jovens que necessitem de atenção à saúde mental (MATSUKURA; FERNANDES; CID, 2014).

Diante da necessidade de se conhecer melhor a relação entre a saúde mental das crianças e seus níveis de atividade física, com o propósito de planejar intervenções futuras aliadas à escola, família e comunidade, estudos de caráter descritivo que visem ao levantamento de dados e geração de possíveis hipóteses são fundamentais ao campo de pesquisa.

Em síntese, o objetivo do estudo foi verificar a relação entre problemas de saúde mental e os níveis de atividade física em crianças escolares da cidade de Jacobina-Ba.

\section{Método}

\subsection{Sujeitos}

Os sujeitos da pesquisa foram escolares de 8 a 10 anos, de ambos os sexos, matriculados em escolas da Rede Pública Municipal de Jacobina, Bahia, e também seus pais/responsáveis, que foram informantes secundários no fornecimento dos dados sobre a saúde mental de seus filhos. A amostra foi composta por 55 escolares, recrutados por conveniência a partir da autorização e disponibilidade dos pais/responsáveis em querer participar do estudo.

\subsection{Local}

O estudo foi realizado em escolas municipais da cidade de Jacobina, Bahia, Brasil. Segundo o Censo de 2010, a cidade de Jacobina possui 79.247 habitantes, e, dentre esses, um total de 25.891 pessoas que frequentam creches ou escolas. Jacobina fica localizada na região do Piemonte da Diamantina e sua principal renda é o comércio e a mineração (INSTITUTO..., 2012).

A ideia inicial era realizar um levantamento em toda a rede municipal de ensino. Para isso, recorreu-se à Secretaria de Educação local, onde os dados de matrículas e a quantidade e distribuição das escolas foram fornecidos. Ao todo foram localizadas 12 escolas na zona urbana do município que possuíam matrículas de alunos na faixa etária delimitada para o estudo.

Foram feitas visitas a todas as escolas e agendadas reuniōes com os pais das crianças, em dias e horários previamente combinados com a direção e coordenação de cada escola. Devido ao insucesso dessas reunióes, já que poucos pais compareciam, foram feitas tentativas de reunióes com os pais das crianças diretamente nos domicílios, com auxílio de agentes de saúde da região, que facilitavam a localização das residências. Mesmo diante dessas estratégias, a maioria dos pais não aceitou participar do estudo, já que de um total de 275 crianças potencialmente alvo do estudo, a partir dos dados de matrículas, somente 150 foram localizadas e, desse total, apenas 55 pais/responsáveis aceitaram participar do estudo.

\subsection{Instrumentos}

Foi aplicado o questionário Child Behavior Checklist (CBCL) para avaliação dos problemas de saúde mental dos escolares e o Physical Activity Questionaire for Older Children (PAQ-C), para avaliação dos níveis de atividade física dos sujeitos. Previamente à aplicação desses instrumentos, recolheram-se informaçóes relativas aos dados pessoais dos sujeitos através de um formulário construído para o estudo.

$\mathrm{O}$ CBCL, na sua versão brasileira, denomina-se "Inventário de Comportamento da Infância e Adolescência", avalia os problemas emocionais e de comportamento em crianças e adolescentes de 4 a 18 anos, a partir de informaçóes fornecidas pelos pais ou responsáveis (LIMA, 2010). O instrumento é composto por 138 sentenças, nas quais 118 referem-se a problemas de comportamento e 20 à competência social (WIELEWICKI et al., 2011). A cada um dos itens é atribuído uma pontuação, a saber: 0 (não verdadeiro), 1 (pouco verdadeiro) e 2 (muito verdadeiro) (ACHENBACH, 2001). O CBCL possui um total de 11 escalas que permitem identificar problemas sociais, problemas de pensamento e atenção, problemas externalizantes e problemas internalizantes. Esse rol de escalas permite uma classificação geral, denominada de total de problemas clínicos/problemas de saúde mental, que permite classificar a criança em clínica ou não clínica e é muito utilizada em estudos de rastreamento (SANTOS; SILVARES, 2006; LIMA, 2010). No presente estudo foi adotado o ponto de corte $\mathrm{T}>63$ para a classificação de problemas de saúde mental (LIMA, 2010) e a nomenclatura Transtorno Mental Positivo (crianças clínicas) e Negativo (crianças não clínicas) na distribuição das crianças da amostra deste estudo. Os dados preliminares do estudo de validação desse 
instrumento apresentou uma sensibilidade de $80,4 \%$ na aplicação com mães com baixa escolaridade (BORDIN; MARI; CAEIRO, 1995), o que permite inferir que o instrumento é adequado para pesquisas de campo que objetivem rastrear problemas mentais em crianças na idade escolar.

O PAQ-C foi desenvolvido por Crocker et al. (1997), cujo estudo de validação revela valores de consistência interna entre 0,79 e 0,89 e valores de teste-reteste entre 0,75 e 0,82 . O instrumento foi traduzido e modificado para a língua portuguesa, apenas para incluir atividades físicas e esportivas praticadas no Brasil (SILVA; MALINA, 2000). Este avalia o nível de atividades físicas moderadas e intensas de crianças e adolescentes nos sete dias anteriores ao preenchimento do questionário. Esse questionário é composto por 9 questóes sobre a prática de esportes e jogos, atividades físicas na escola e lazer, incluindo o final de semana. Cada questão tem valor de $1 \mathrm{a} 5$, e o escore final é obtido pela média das questôes. Os escores são representados por muito sedentário (1), sedentário (2), moderadamente ativo (3), ativo (4) e muito ativo (5). Para fins de análise, as classificaçóes foram dicotomizadas em Sedentários e Ativos (Ativos + Moderadamente Ativos), como já vem sendo usado na literatura, a fim de facilitar as análises estatísticas (RIVERA et al., 2010).

\subsection{Procedimentos}

A coleta de dados foi composta por dois avaliadores estudantes de Educação Física, que realizaram as mesmas funçóes durante todo o processo de coleta dos dados. As coletas foram iniciadas em 23 de fevereiro de 2015 e concluídas em 19 de junho de 2015. Foram feitas reuniōes de sensibilização com os pais nas escolas, nas quais as coletas eram previamente agendadas. Nas coletas de dados, os país/responsáveis autorizavam a participação das crianças e adolescentes por meio da assinatura do Termo de Consentimento Livre e Esclarecido (TCLE), depois eles respondiam os questionários propostos para os estudantes recrutados, como informante secundário desses. Após a coleta de dados, para fazer a análise descritiva dos dados, foi utilizado um suporte para o tratamento estatístico. E através dos resultados fizemos análise do estudo.

\subsection{Análise estatística}

Foram feitas análises estatísticas descritivas, com distribuição de frequências relativas e absolutas, médias e desvios-padrão. Para verificar a associação entre os transtornos mentais e níveis de atividade física, e a associação dessas variáveis com os fatores sociodemográficos, recorreu-se ao teste do qui-quadrado com nível de significância $\mathrm{p}<0,05$.

Utilizou-se ainda a medida de razão de chances (OR) com intervalo de confiança de 95\%. Todas as análises foram feitas no pacote estatístico SPSS ${ }^{\circledR}$ (Statistical Package for the Social Sciences), versão 20.0 .

\subsection{Aspectos éticos}

A pesquisa seguiu todos os preceitos da ética em pesquisa com seres humanos da Declaração de Helsinque e da Resoluçáo do Conselho Nacional de Saúde/Ministério da Saúde (466/12), tendo sido aprovada pelo Comitê de Ética em Pesquisa com Seres Humanos da Universidade do Estado da Bahia (UNEB), com número CAEE 30168914.5.0000.0057. Todos os responsáveis pelos sujeitos voluntários da pesquisa assinaram o Termo de Consentimento Livre e Esclarecido (TCLE).

\section{Resultados}

A média de idade das crianças foi de 9,20 anos $( \pm 0,80 \mathrm{DP})$, já a média de idade das mães das crianças foi de 35,27 anos ( $\pm 8,33 \mathrm{DP})$. A média de idade das meninas foi de 9,15 anos $( \pm 0,79 \mathrm{DP})$ e dos meninos de 9,27 anos $( \pm 0,82 \mathrm{DP})$.

A Tabela 1 expõe a caracterização, em frequências relativas e absolutas, dos informantes da amostra do estudo por meio das principais variáveis investigadas na pesquisa.

A Tabela 2 expóe a caracterização, em frequências relativas e absolutas, das crianças da amostra do estudo por meio das principais variáveis investigadas

Tabela 1. Caracterização dos informantes do estudo $(\mathrm{N}=55)$.

\begin{tabular}{lcc}
\hline \multicolumn{1}{c}{ Variável } & $\mathbf{n}$ & $\mathbf{\%}$ \\
\hline Estado civil materno & & \\
Solteira & 28 & 50,9 \\
Casada & 20 & 36,4 \\
Separada & 07 & 12,7 \\
Escolaridade materna & & \\
$\leq 8$ anos de estudo & 38 & 69,1 \\
$>8$ anos de estudo & 17 & 30,9 \\
Atividade laboral materna & & \\
Trabalha & 38 & 69,1 \\
Não trabalha & 17 & 30,9 \\
Recebe benefício do governo & & \\
Sim & 41 & 74,5 \\
Não & 14 & 25,5 \\
\hline
\end{tabular}


na pesquisa. Cabe destacar que a maioria das crianças era sedentária (80\%) e que apenas $07,3 \%$ da amostra apresentou rastreamento positivo para transtorno mental.

A Tabela 3 expóe a análise de associação entre o transtorno mental e os níveis de atividade física.

Tabela 2. Caracterização das crianças do estudo $(\mathrm{N}=55)$.

\begin{tabular}{lcc}
\hline \multicolumn{1}{c}{ Variável } & $\mathbf{n}$ & $\%$ \\
\hline Sexo & & \\
Masculino & 22 & 40,0 \\
Feminino & 33 & 60,0 \\
A criança reside com pai biológico & & \\
Sim & 39 & 70,9 \\
Não & 16 & 29,1 \\
Níveis de atividade física & & \\
Ativos & 11 & 20,0 \\
Sedentários & 44 & 80,0 \\
Transtorno Mental & & \\
Positivo & 04 & 07,3 \\
Negativo & 51 & 92,7 \\
\hline
\end{tabular}

Apesar de a maioria das crianças com classificação positiva para transtorno mental ser sedentária (75\%), não foi encontrada associação significativa entre o grupo.

A Tabela 4 evidencia a análise de associação entre os níveis de atividade física das crianças, avaliados por meio do PAQ-C, e as variáveis sociais e familiares investigadas no presente estudo. Dentre as variáveis, a única que apresentou associação com os níveis de atividade física foi o sexo, uma vez que a maioria das meninas foi classificada como sedentária $(93,9 \%)$, quando comparadas aos meninos (59,1\%), com diferença significativa $(\mathrm{p}<0,01)$. Verificou-se, ainda, que as meninas apresentaram um risco 10 vezes superior de serem sedentárias quando comparadas aos meninos.

Ao se verificar possíveis associaçóes entre o transtorno mental e as variáveis sociais e familiares investigadas no presente estudo, não foram observadas diferenças significativas na amostra de crianças escolares participantes desta pesquisa.

Tabela 3. Análise da associação entre o transtorno mental e os níveis de atividade física das crianças participantes do estudo $(\mathrm{N}=55)$.

\begin{tabular}{|c|c|c|c|c|}
\hline \multirow{2}{*}{ Transtorno Mental } & \multicolumn{2}{|c|}{ Níveis de atividade física } & \multirow{2}{*}{ p-valor* } & \multirow{2}{*}{ OR (IC: 95\%) } \\
\hline & Ativos & Sedentários & & \\
\hline Positivo & $01(25,0 \%)$ & $03(75,0 \%)$ & \multirow{2}{*}{0,79} & \multirow{2}{*}{$0,73(0,06-7,79)$} \\
\hline Negativo & $10(19,6 \%)$ & $41(80,4 \%)$ & & \\
\hline
\end{tabular}

*Nível de significância $\mathrm{p}<0,05$ com a utilização do qui-quadrado.

Tabela 4. Análise da associação entre os níveis de atividade física das crianças e as variáveis sociais e familiares investigadas no estudo $(\mathrm{N}=55)$.

\begin{tabular}{|c|c|c|c|c|}
\hline \multirow{2}{*}{ Variáveis } & \multicolumn{2}{|c|}{ Níveis de atividade física } & \multirow{2}{*}{ p-valor* } & \multirow{2}{*}{ OR (IC: 95\%) } \\
\hline & Ativos & Sedentários & & \\
\hline \multicolumn{5}{|l|}{ Sexo } \\
\hline Masculino & $09(40,9)$ & $13(59,1)$ & $<0,01 *$ & $10,73(2,03-56,63)$ \\
\hline Feminino & $02(06,1)$ & $31(93,9)$ & & \\
\hline \multicolumn{5}{|l|}{ Estado civil materno } \\
\hline Solteira/Separada & $07(20,0)$ & $28(80,0)$ & 1,00 & $1,00(0,25-3,94)$ \\
\hline Casada & $04(20,0)$ & $16(80,0)$ & & \\
\hline \multicolumn{5}{|l|}{ Escolaridade materna } \\
\hline$\leq 8$ anos de estudo & $07(18,4)$ & $31(81,6)$ & 0,66 & $1,36(0,34-5,46)$ \\
\hline$>8$ anos de estudo & $04(23,5)$ & $13(76,5)$ & & \\
\hline \multicolumn{5}{|c|}{ A criança reside com o pai biológico } \\
\hline Sim & $09(23,1)$ & $30(76,9)$ & 0,38 & $2,10(0,40-11,02)$ \\
\hline Não & $02(12,5)$ & $14(87,5)$ & & \\
\hline \multicolumn{5}{|c|}{ Atividade laboral materna } \\
\hline Trabalha & $09(23,7)$ & $29(76,3)$ & 0,31 & $2,32(0,44-12,17)$ \\
\hline Não trabalha & $02(11,8)$ & $15(88,2)$ & & \\
\hline \multicolumn{5}{|c|}{ Recebe benefício do governo } \\
\hline Sim & $07(17,1)$ & $34(82,9)$ & 0,35 & $0,51(0,12-2,12)$ \\
\hline Não & $04(28,6)$ & $10(71,4)$ & & \\
\hline
\end{tabular}

*Nível de significância $\mathrm{p}<0,05$ com a utilização do qui-quadrado. 


\section{Discussão}

O presente estudo identificou uma prevalência de 07,3\% de problemas de saúde mental nas crianças investigadas. Apesar da baixa prevalência, estudos nacionais têm encontrado prevalências superiores a $12 \%$ em crianças em idade escolar (ANSELMI et al., 2010, 2004; FLEITLICH-BILYK; GOODMAN, 2004). Isso permite inferir que contextos regionais específicos, como o do presente estudo, podem fornecer aspectos favoráveis à saúde mental dessas crianças, aspectos esses que precisam ser investigados, por exemplo, as relaçóes familiares, visto que os níveis de atividade física, considerados aqui como a principal variável protetora investigada, não esteve associado à saúde mental das crianças.

Apesar de a maioria das crianças com rastreamento positivo para transtorno mental ter sido classificada como sedentária, não houve associação entre a saúde mental e os níveis de atividade física das crianças ao nível de $\mathrm{p}<0,05$. Além disso, observou-se ainda que as meninas apresentaram um risco 10 vezes superior de serem sedentárias quando comparadas aos meninos.

Pode-se fazer comparação entre este estudo e a pesquisa de Novaes (2005), em que foram recrutadas 245 crianças, de 9 a 12 anos, que frequentaram escolas públicas do município de Americana-SP em 2003, e foram feitas intervençóes com atividades de Educação Física e Arte-Educação nos grupos estudados. Nesse estudo foi observado que as intervençôes através de atividades lúdicas possibilitam uma diminuição no desenvolvimento de problemas de saúde mental e um aumento nos fatores de proteçáo em um período curto de intervenção, o que aumenta a possibilidade dessa intervenção promover resultados preventivos em longo prazo.

Ainda no estudo de Novaes (2005), o grupo de alunos que apresentavam alto risco severo de problemas de saúde mental passou para alto risco moderado e baixo risco, e apresentaram reduçáo significativa de externalização e internalização, porém os fatores de proteção em saúde mental não aumentaram significativamente. As intervençóes com a arteterapia apresentaram apenas um efeito terapêutico, não conferindo prevenção para futuros transtornos mentais. Já o grupo de arte-capoeira e arte-educação promoveu um suporte social adequado e aumentou os fatores de proteçáo.

Isso leva a crer que, mesmo diante da não associação encontrada no presente estudo, o papel da atividade física na vida das crianças é indiscutível, sobretudo por evidências de que sua prática regular é um fator de proteção importante ao acometimento de desordens de cunho mental nas crianças.

No estudo realizado por Costa e Assis (2010), numa amostra de 2936 escolares, da rede pública e privada da cidade de Florianópolis-SC, encontrou-se um resultado também semelhante a esta pesquisa, no qual, também, as meninas foram mais sedentárias que os meninos. Tal fato pode ser devido à menor participação das meninas em atividades esportivas, diferente dos meninos, que são mais incentivados às práticas esportivas, tais como jogar bola, fazer ginástica, andar de skate, como relatam Costa e Assis (2010).

É possível, ainda, fazer comparaçôes entre os dados do presente estudo com a pesquisa de Merege Filho et al. (2013). Em sua pesquisa foi investigada a associação da atividade física de lazer com o desempenho cognitivo em crianças saudáveis. Nesse estudo participaram 100 crianças de 10 a 12 anos de idade, de ambos os sexos. Todos os participantes foram recrutados de uma mesma escola da rede pública de ensino da cidade de São Paulo-SP. Nesse estudo, as crianças classificadas como ativas não atingiram um nível de atividade física de lazer tal que pudesse se refletir em melhora do desempenho cognitivo geral. E as crianças classificadas como insuficientemente ativas alcançaram um nível de atividade física suficientemente adequada, a ponto de promoverem desempenho cognitivo comparável a de seus pares considerados ativos.

Outro estudo que colaborou com os achados desta foi a pesquisa de Sá et al. (2010), a qual teve o objetivo de identificar fatores associados a problemas de saúde mental em crianças/adolescentes em amostra probabilística de 67 sujeitos na faixa etária 4-17 anos, realizado numa comunidade urbana de baixa renda do município de Embu-SP. Foi apontado em seus resultados que os principais fatores associados aos problemas de saúde mental em crianças são os seguintes: ser criança do sexo masculino; sofrer punição física grave; ideação suicida da mãe; violência conjugal física grave contra a mãe; e embriaguez do pai ou padrasto. As taxas identificadas para os problemas de saúde mental, segundo CBCL, foram as seguintes: $19,4 \%$ de problemas do tipo internalização, $20,9 \%$ de problemas do tipo externalização. No presente estudo, utilizou-se apenas o escore total do CBCL para problemas mentais.

Ainda no estudo de Sá et al. (2010) observou-se que crianças com idade entre 4 e 10 anos têm maior número de casos de problemas mentais do tipo externalização. Da mesma forma, crianças que sofrem violência física grave também apresentam mais casos de problemas de saúde mental do tipo externalização. 
Já os problemas de internalização estiveram associados à embriaguez do pai ou padrasto, e o gênero das crianças também foi um fator determinante, uma vez que os meninos apresentaram mais problemas de internalização.

O estudo de Matsukura, Fernandes e Cid (2014), que procurou identificar e comparar situaçôes de risco ou proteção ao desenvolvimento socioemocional de crianças que vivem em contextos de desvantagem socioeconômica, no estado de São Paulo, numa amostra de 7 crianças, encontrou resultados diferentes ao de Sá et al. (2010), pois a condição socioeconômica desfavorável, a baixa escolaridade dos responsáveis, a presença de discussôes na família e a prática de bater e castigar os filhos não foram apontados como fatores de risco à saúde mental infantil.

Outro estudo em que também houve a associação de problemas mentais com os fatores sociais, realizado nos Estados Unidos, examinou o temperamento da criança, o comportamento materno, os efeitos de suas interaçóes com outras pessoas e o funcionamento social da criança. Os sujeitos da pesquisa foram recrutados em um ambulatório de base universitária clínica a partir de uma revisão dos registros clínicos de pediatras e psiquiatras. A amostra final foi composta por 474 crianças de 10 anos de idade, sendo 207 famílias. Nesse estudo, identificou-se que o comportamento inibitório caracterizado pela timidez, preocupação e fadiga (HA) está correlacionado com o funcionamento social das crianças (BAER et al., 2015).

Ainda no estudo de Baer et al. (2015), as crianças cujas mães eram classificadas com maior tendência a procurar experiências estimulantes, impulsividade, extravagância e desordem, ou comportamento inibitório caracterizado pela timidez, preocupação e fadiga, também apresentaram mais problemas sociais. Da mesma forma, a criança que apresentou níveis mais baixos de capacidade para perseverar, apesar de frustração, cansaço e reforço intermitente, teve mais problemas sociais e baixa competência social (BAER et al., 2015).

Da mesma forma, no estudo de Hallal et al. (2008), realizado na cidade de Pelotas/RS, foi avaliada a influência de variáveis psicológicas dos primeiros anos de vida sobre o nível de atividade física e desempenho motor aos 4 anos de idade, sendo selecionadas 634 crianças participantes de uma coorte de nascimento. Através da escala do CBCL, foi utilizado apenas o escore total e a síndrome retração/depressão. Fizeram essa opção com o objetivo de comparar crianças com facilidade ou dificuldade de interação com outras crianças em termos de atividade física.
No estudo de Hallal et al. (2008), os principais achados foram que as crianças com bom relacionamento com seus pares foram mais ativas e tiveram melhor desempenho motor em comparação às crianças classificadas como tendo uma relação ruim com outras crianças. Esses achados reforçam a importância das relaçôes com os pares na vida social de crianças, desde a idade pré-escolar. E resultados similares foram encontrados para aqueles com problemas comportamentais, particularmente retração/depressão.

O estudo de Hernández et al. (2011) também colabora com nossos achados, uma vez que foi avaliada a associação entre sedentarismo, obesidade e saúde mental de crianças escolares, numa amostra composta por 9.122 crianças, na Espanha, e mostrou que os indivíduos mais sedentários, em relação aos não sedentários, apresentavam mais frequentemente problemas emocionais, problemas de conduta, problemas de relacionamento e dificuldades de relação social. Vale ressaltar que aqueles que praticavam atividade física de lazer apresentaram menor prevalência de problemas de saúde mental.

Martikainen et al. (2012), que avaliaram o nível de atividade física e a saúde mental de crianças em Helsinque, Finlândia, numa amostra de 321 participantes, em seus resultados mostraram que as crianças que tiveram um maior tempo gasto em atividade física geral apresentam menores chances de ter problemas emocionais, como ansiedade e depressão, e também problemas de exclusão social e de comportamento. Logo, o tempo gasto em atividade física esteve associado com chances mais baixas de se expor a problemas de saúde mental. Ou, ainda, que baixos índices de problemas de saúde mental seriam mais característicos em crianças que passaram mais tempo praticando atividade física intensa.

Tubić e Dordić (2013) analisaram o efeito do exercício físico sobre a saúde mental de crianças pré-escolares na República da Sérvia, em 184 crianças, e concluíram que houve uma reduçáo nos indicadores do comportamento de externalizaçáo no grupo de crianças em relaçáo ao início e ao final do programa de intervenção. Além disso, o programa de intervenção teve efeitos mais fracos sobre os indicadores do comportamento de internalização. Assim, percebe-se que é necessário ter um programa de intervenção de atividade física regular e contínua, para ter benefícios na saúde mental das crianças. Os resultados indicam, ainda, que o exercício físico teve influência sobre a redução de problemas de comportamento.

Outro estudo que contribuiu com os achados deste estudo foi o de Vella et al. (2015), que avaliou as associaçóes entre a prática esportiva e o desenvolvimento de forças psicológicas e dificuldades 
durante a infância, numa amostra de 4042 crianças, na Austrália, e comprovaram que as crianças que mantiveram a participação no desporto tiveram menores taxas de problemas de saúde mental em comparação com as crianças que abandonaram o esporte, segundo o relato dos pais. Os pais também relataram que as crianças que participaram de esportes organizados tinham menos problemas de internalização em comparação às crianças que abandonaram os esportes e às crianças que não participavam de nenhum esporte. Essas relaçóes náo tiveram diferenças nas variáveis do estudo, em relação ao IMC, situação socioeconômica ou educação parental. Desse modo, considera-se que é de suma importância o incentivo e a implementação de programas esportivos para garantir benefícios para a saúde mental de crianças.

Em relação ao que foi discorrido, algumas limitaçóes devem ser apontadas. Começando pelo pequeno tamanho da amostra, fato que reduziu o poder estatístico da análise, consequentemente, alguns dados devem ser interpretados com cautela. $\mathrm{O}$ pequeno tamanho amostral deveu-se à dificuldade encontrada para que os pais/responsáveis das crianças se disponibilizassem a participar do estudo. Houve diversas perdas devido ao não comparecimento deles nos dias e horários agendados para a reuniáo de coleta de dados, e ainda falta de sensibilizaçáo com a pesquisa. Além disso, muitos pais/responsáveis se negaram a autorizar a participação do filho no estudo, por não compreenderem a importância da pesquisa científica para a escola e principalmente para seu filho, mesmo tal questão tendo sido claramente exposta pelos pesquisadores e coordenação pedagógica da escola.

Isso leva a questionar o fato de que pais interessados e mais participativos na escola de seus filhos podem implicar pais mais presentes na realidade escolar e no dia a dia dos filhos, o que poderia refletir em melhores condiçóes de saúde das crianças, como maiores níveis de atividade física e melhores condiçốes de saúde mental. Contudo, são necessários mais estudos que possam explorar essas questôes e confirmar essas inquietaçóes.

\section{Conclusão}

Náo se observou associação entre problemas de saúde mental e níveis de atividade física entre as crianças escolares participantes deste estudo. Apesar disso, houve um elevado percentual de crianças consideradas sedentárias e que necessitam de programas de intervenção com atividade física. Para tanto, família e escola devem ser aliadas na melhoria dos indicadores de saúde, adotando atitudes condizentes com hábitos saudáveis de vida, que devem se iniciar na infância.

Apesar da baixa prevalência de problemas de saúde mental encontrada entre as crianças, não se deve desconsiderar esses resultados, uma vez que tais problemas demandam uma maior atenção por parte do corpo pedagógico da escola e necessitam de intervençóes direcionadas em parceria com os sistemas de saúde e educação do município.

Sáo necessários mais estudos que possam retratar com amostras maiores o diagnóstico do perfil de atividade física e saúde mental de crianças escolares. Para tanto, diferentes estratégias devem ser adotadas para que a participação dos pais/responsáveis aconteça, uma vez que, para a realizaçáo de futuras intervençốes, a participação familiar é primordial.

\section{Referências}

ACHENBACH, T. M. Manual for the child behavior checklist/6-18 and 2001 profile. Burlington: University of Vermont, 2001.

ANSELMI, L. et al. Psychosocial determinants of behaviour problems in Brazilian preschool children. Journal of Child Psychology and Psychiatry, Elmsford, v. 45, n. 4, p. 779-788, 2004.

ANSELMI, L. et al. Prevalence of psychiatric disorders in a Brazilian birth cohort of 11-year-olds. Social Psychiatry and Psychiatric Epidemiology, Berlin, v. 45, n. 1, p. 135-142, 2010.

ASBAHR, F. R.; TIWARI, S.; KENDALL, P. C. Transtornos da ansiedade na infância e adolescência. Rio de Janeiro: Elsevier, 2007.

AVANCI, J. Q.; ASSIS, S. G.; PESCE, R. P. Depressāo em crianças: uma reflexão sobre crescer em meio à violência. Rio de Janeiro: FIOCRUZ/ENSP/CLAVES/CNPq, 2008. Disponível em: <http://www5.ensp.fiocruz.br/ biblioteca/dados/txt_720672695.pdf>. Acesso em: 03 ago. 2015 .

BAER, J. et al. Child temperament, maternal parenting behavior, and child social functioning. Journal of Child and Family Studies, New York, v. 24, n. 4, p. 1152-1162, 2015.

BORDIN, I. A. S.; MARI, J. J.; CAEIRO, M. F. Validação da versão brasileira do "Child Behavior Checklist" (CBCL) (Inventário de Comportamentos da Infância e Adolescência): dados preliminares. Revista Brasileira de Psiquiatria, São Paulo, v. 2, n. 17, p. 55-66, 1995.

CONNOLLY, S. D.; BERNSTEIN, G. A. Practice parameter for the assessment and treatment of children and adolescents with anxiety disorders. Journal of the American Academy of Child and Adolescent Psychiatry, Baltimore, v. 46, n. 2, p. 267-283, 2007. 
COSTA, F. F.; ASSIS, M. A. A. Nível de atividade física e comportamentos sedentários de escolares de sete a dez anos de Florianópolis-SC. Revista Brasileira de Atividade Física e Saúde, Florianópolis, v. 16, n. 1, p. 48-54, 2010.

CROCKER, P. R. et al. Measuring general levels of physical activity: preliminary evidence for the Physical Activity Questionnaire for Older Children. Medicine and Science in Sports and Exercise, Madison, v. 29, n. 10, p. 1344-1349, 1997.

FLEITLICH-BILYK, B.; GOODMAN, R. Prevalence of child and adolescent psychiatric disorders in Southeast Brazil. Journal of the American Academy of Child and Adolescent Psychiatry, Baltimore, v. 43, n. 6, p. 727-734, 2004.

HALLAL, P. C.; ANSELMI, L.; AZEVEDO, M. Influência de variáveis psicológicas e da infância sobre os níveis de atividade física e desempenho motor aos quatro anos de idade. Revista Brasileira de Atividade Física \& Saúde, Florianópolis, v. 13, n. 2, p. 65-74, 2008.

HALPERN, R.; FIGUEIRAS, A. C. M. Influências ambientais na saúde mental da criança. Jornal de Pediatria, Rio de Janeiro, v. 80, n. 2, p. 104-110, 2004.

HERNÁNDEZ, A. R. et al. Inactivity, obesity and mental health in the spanish population from 4 to 15 years of age. Revista Espanhola de Salud Pública, Madrid, v. 85, n. 4, p. 373-382, 2011.

INSTITUTO BRASILEIRO DE GEOGRAFIA E ESTATÍSTICA - IBGE. Censo demográfico 2010. Rio de Janeiro, 2012.

LIMA, F. M. Fatores de risco precoces para problemas emocionais e de comportamento em crianças e pré-adolescentes. 2009. 66 f. Dissertação (Mestrado em Ciências Médicas) - Universidade Federal do Rio Grande do Sul, Porto Alegre, 2010.

MARTIKAINEN, S. et al. Physical activity and psychiatric problems in children. The Journal of Pediatrics, St. Louis, v. 161, n. 1, p. 160-164, 2012.

MATSUKURA, T. S.; FERNANDES, A. D. S. A.; CID, M. F. B. Saúde mental infantil em contextos de desvantagem socioeconômica: fatores de risco e proteção. Cadernos de Terapia Ocupacional da UFSCar, São Carlos, v. 22, n. 2, p. 251-262, 2014.

MEARS, R.; JAGO, R. Effectiveness of after-school interventions at increasing moderate-to-vigorousphysical activity levels in 5- to 18-year olds: a systematic review and meta-analysis. British Journal of Sports Medicine, Loughborough, v. 50, p. 1315-1324, 2016. http:// dx.doi.org/10.1136/bjsports-2015-094976.

MEREGE FILHO, C. A. A. et al. Associação entre o nível de atividade física de lazer e o desempenho cognitivo em crianças saudáveis. Revista Brasileira de Educação Física e Esporte, São Paulo, v. 27, n. 3, p. 355-361, 2013.

NOVAES, J. J. Suporte social como prevenção em saúde mental e obesidade: Intervençóes através de atividades lú- dicas no cenário escolar. 2005. 359 f. Tese (Doutorado em Saúde da Criança e do Adolescente) - Universidade de Campinas, Campinas, 2005.

ORGANIZAÇÃO MUNDIAL DA SAÚDE - OMS. Relatório sobre a saúde no mundo 2001. Saúde Mental: nova concepção, nova esperança. Genebra: OPAS/ OMS, 2001.

RAMIRES, V. R. R. et al. Saúde mental de crianças no brasil: uma revisão de literatura. Revista Interação em Psicologia, Curitiba, v. 13, n. 2, p. 311-322, 2009 a.

RAMIRES, V. R. R. et al. Fatores de risco e problemas de saúde mental de crianças. Arquivos Brasileiros de Psicologia, Rio de Janeiro, v. 61, n. 2, p.1-14, 2009b.

RIVERA, I. R. et al. Atividade física, horas de assistência à TV e composição corporal em crianças e adolescentes. Arquivos Brasileiros de Cardiologia, São Paulo, v. 95, n. 2, p. 159-165, 2010.

SÁ, D. G. F. et al. Fatores de risco para problemas de saúde mental na infância/adolescência. Psicologia: Teoria e Pesquisa, Sáo Paulo, v. 26, n. 4, p. 643-652, 2010.

SANTOS, E. O. L.; SILVARES, E. F. M. Crianças enuréticas e crianças encaminhadas para clínicas-escola: um estudo comparativo da percepção de seus pais. Psicologia: Reflexão e Crítica, Porto Alegre, v. 19, n. 2, p. 277-282, 2006.

SILVA, P. V. C.; COSTA, J. Á. L. Efeitos da atividade física para a saúde de crianças e adolescentes. Revista Psicologia Argumento, Curitiba, v. 29, n. 64, p. 41-50, 2011. SILVA, R. C. R.; MALINA, R. M. Nível de atividade física em adolescentes do Município de Niterói, Rio de Janeiro. Cadernos de Saúde Pública, Rio de Janeiro, v. 16, n. 4, p. 1091-1097, 2000.

TUBIĆ, T.; DORDIĆ, V. Exercise effects on mental health of preschool children. Anales de Psicologia, Murcia, v. 29, n. 1, p. 249-256, 2013.

VELLA, S. A. et al. Associations between sports participation and psychological difficulties during childhood: a two-year follow up. Journal of Science and Medicine in Sport, Belconnen, v. 18, n. 3, p. 304-309, 2015.

WIELEWICKI, A.; GALLO, A. E.; GROSSI, R. Instrumentos na prática clínica: CBCL como facilitador da análise funcional e do planejamento da intervenção. Temas em Psicologia, Ribeirão Preto, v. 19, n. 2, p. 513 523, 2011.

XIMENES, L. F.; PESCE, R. P. Problemas de saúde mental em crianças: abordagem na atenção básica. Ciência \& Saúde Coletiva, São Paulo, v. 14, n. 2, p. 671-672, 2009. 


\section{Contribuição dos Autores}

Gabrielle - realizou a concepção do texto, organização e coleta dos dados e redação do texto. Jorge - realizou a concepçáo do texto, orientou toda a condução do trabalho, analisou os dados, realizou as análises estatísticas e redação do texto. Ambos aprovaram a versão final do texto.

\section{Fonte de Financiamento}

O trabalho foi financiado pela Fundação de Amparo à Pesquisa do Estado da Bahia (FAPESB). 